\title{
Ulam-Hyers stability, well-posedness and limit shadowing property of the fixed point problems for some contractive mappings in $M_{s}$-metric spaces
}

\author{
Mi Zhou ${ }^{a}$, Xiao-lan Liü,c,*, Yeol Je Cho ${ }^{\text {d,e,*, }}$, Boško Damjanovićf \\ a School of Science and Technology, Sanya College, 572022, Sanya, Hainan, China. \\ ${ }^{b}$ College of Science, Sichuan University of Science and Engineering, 643000, Zigong, Sichuan, China. \\ ${ }^{c}$ Sichuan Province University Key Laboratory of Bridge Non-destruction Detecting and Engineering Computing, Zigong, Sichuan \\ 643000, China. \\ ${ }^{d}$ Department of Mathematics Education, Gyeongsang National University, Jinju 660-701, Korea. \\ ${ }^{e}$ Center for General Education, China Medical University, Taichung 40402, Taiwan. \\ ${ }^{f}$ Faculty of Agriculture, University of Belgrade, Belgrade, Serbia.
}

Communicated by P. Kumam

\begin{abstract}
In this paper, first, we introduce several types of the Ulam-Hyers stability, the well-posedness and the limit shadowing property of fixed point problems in $M_{s}$-metric spaces. Second, we give such results for fixed point problems of Banach and Kannan contractive mappings in $M_{s}$-metric spaces. Finally, we give some examples to illustrate the validity of our main results. (C)2017 All rights reserved.
\end{abstract}

Keywords: Fixed point problem, Ulam-Hyers stability, well-posedness, limit shadowing property, $M_{s}$-metric spaces. 2010 MSC: 47H09, 47H10, 54H25.

\section{Introduction and preliminaries}

In 1922, Banach [1] proved a famous theorem, which is well-known as "Banach's contraction principle" or "Banach's fixed point theorem", to establish the existence of solutions for integral equations.

Theorem $1.1([1])$. Let $(\mathrm{X}, \mathrm{d})$ be a complete metric space and $\mathrm{T}: \mathrm{X} \mapsto \mathrm{X}$ be a contractive mapping, that is, there exists $\mathrm{k} \in[0,1)$ such that

$$
d(T x, T y) \leqslant k d(x, y),
$$

for all $x, y \in X$. Then $T$ has a unique fixed point $z \in X$, that is, $T z=z$. Furthermore, for each $x_{0} \in X$, the sequence $\left\{x_{n}\right\}$ defined by

$$
x_{n+1}=T x_{n}
$$

for all $\mathrm{n} \geqslant 0$ converges to the fixed point $z$ of $\mathrm{T}$.

\footnotetext{
*Corresponding author

Email addresses: mizhou330@126.com (Mi Zhou), stellalwp@163.com (Xiao-lan Liu), yjcho@gnu.ac.kr (Yeol Je Cho), dambo@agrif.bg.ac.rs (Boško Damjanović)

doi:10.22436/jnsa.010.05.02
} 
Since then, because of its simplicity, usefulness and applications, it has become a very popular and useful tool in solving the existence problems in many branches of mathematical analysis.

From Banach's contraction principle, it follows that the contractive mapping is continuous. It is inspired to consider that do there exist some contractive conditions which need the mapping $T$ has not to be necessarily continuous?

The answer for this inquiry was provided by Kannan [5] in 1969 who proved "Kannan's fixed point theorem" for the following contractive mapping, which is called Kannan's contraction.

Theorem $1.2([5])$. Let $(\mathrm{X}, \mathrm{d})$ be a complete metric space and $\mathrm{T}: \mathrm{X} \mapsto \mathrm{X}$ be a contractive mapping, that is, there exists $\mathrm{k} \in\left[0, \frac{1}{2}\right)$ such that

$$
d(T x, T y) \leqslant k[d(x, T x)+d(y, T y)]
$$

for all $x, y \in X$. Then, $T$ has a unique fixed point $z \in X$, that is, $\mathrm{T} z=z$.

In 1975, Subrahmanyam [12] proved that Kannan's contraction characterizes the metric completeness, that is, if every Kannan's contractive mapping has a fixed point, then a metric space $(X, d)$ is complete.

Recently, some authors have introduced some extensions of metric spaces in several ways and studied fixed point theory and applications in these metric spaces.

Especially, in 2012, Sedghi et al. [11] introduced the concept of S-metric spaces which is a space with three dimensions and they studied the topological properties of $S$-metric spaces and showed the existence and uniqueness of a fixed point for certain generalized contractive mappings in the setting of S-metric spaces. In 2014, Mlaiki et al. [7] established an extension of S-metric spaces to partial S-metric spaces and, also, they pointed out that every $S$-metric space is a partial $S$-metric space, but the converse is not true. Recently, the concept of a partial $S$-metric space was extended to the concept of $M_{s}$-space by Mlaiki et al. [8]. Moreover, they showed the existence and uniqueness of a fixed point for a self-mapping in $M_{s}$-metric spaces under Banach's and Kannan's contractive mappings, which are the generalizations of Banach's and Kannan's fixed point theorems in the framework of partial S-metric spaces.

Theorem $1.3([8])$. Let $\left(\mathrm{X}, \mathrm{m}_{\mathrm{s}}\right)$ be a complete $\mathrm{M}_{\mathrm{s}}$-metric space and $\mathrm{T}: \mathrm{X} \mapsto \mathrm{X}$ be a self-mapping on $\mathrm{X}$ satisfying the following condition:

$$
m_{s}(T x, T y, T z) \leqslant k m_{s}(x, y, z),
$$

for all $x, y, z \in X$, where $k \in[0,1)$. Then $T$ has a unique fixed point $u \in X$, such that $m_{s}(u, u, u)=0$.

Theorem 1.4 ([8]). Let $\left(\mathrm{X}, \mathrm{m}_{\mathrm{s}}\right)$ be a complete $\mathrm{M}_{\mathrm{s}}$-metric space and $\mathrm{T}: \mathrm{X} \mapsto \mathrm{X}$ be a self-mapping on $\mathrm{X}$ satisfying the following condition:

$$
m_{s}(T x, T x, T y) \leqslant \lambda\left[m_{s}(x, x, T x)+m_{s}(y, y, T y)\right]
$$

for all $\mathrm{x}, \mathrm{y} \in \mathrm{X}$, where $\lambda \in\left[0, \frac{1}{2}\right)$. Then $\mathrm{T}$ has a unique fixed point $\mathrm{u} \in \mathrm{X}$ such that $\mathrm{m}_{\mathrm{s}}(\mathrm{u}, \mathrm{u}, \mathrm{u})=0$.

On the other hand, recently, some authors studied and extended the Ulam-Hyers stability, the wellposedness and the limit shadowing property of for fixed point problems in various spaces (see $[2-4,6,9$, 10]).

In this paper, we first present various types of the Ulam-Hyers stability, the well-posedness and the limit shadowing property of the fixed point problem in an $M_{s}$-metric space which is an extension of a metric space. Second, we study the Ulam-Hyers stability, the well-posedness and the limit shadowing property of the fixed point problem for Banach's and Kannan's contractive mappings in $M_{s}$-metric spaces. Finally, we give some examples to illustrate the validity of our main results.

Throughout this paper, we denote by $\mathbb{N}, \mathbb{R}^{+}$, and $\mathbb{R}$ the sets of positive integers, nonnegative real numbers, and real numbers, respectively. The following definitions, notations and lemmas are needed in the following discussion. 
Definition 1.5 ([7]). Let $X$ be a nonempty set. A partial $S$-metric on $X$ is a function $S_{p}: X^{3} \mapsto[0, \infty)$ satisfying the following conditions: for all $x, y, z, t \in X$,

$\left(s_{p} 1\right) S_{p}(x, y, z) \geqslant 0$;

$\left(s_{p} 2\right) x=y$ if and only if $s_{p}(x, x, x)=s_{p}(y, y, y)=s_{p}(x, x, y)$

$\left(s_{p} 3\right) s_{p}(x, y, z) \leqslant s_{p}(x, x, t)+s_{p}(y, y, t)+s_{p}(z, z, t)-s_{p}(t, t, t)$;

$\left(s_{p} 4\right) s_{p}(x, x, x) \leqslant s_{p}(x, y, z)$

$\left(s_{p} 5\right) s_{p}(x, x, y)=s_{p}(y, y, x)$.

The pair $\left(X, s_{p}\right)$ is called a partial S-metric space.

Note that every S-metric space is a partial S-metric space, but not every partial S-metric space is an S-metric space.

Now, we give an example to show that a partial S-metric space needs not to be necessarily an S-metric space.

Example 1.6. Let $X=[0,1]$ and define a function $s_{p}: X^{3} \mapsto \mathbb{R}^{+}$by $s_{p}(x, y, z)=\max \{x, y, z\}$ for all $x, y, z \in X$. Then $s_{p}$ is a partial S-metric, but it is not an $S$-metric on $X$. Indeed, for any $0<x<1$, we have $s_{p}(x, x, x)=x \neq 0$.

Next, we present the definition of an $M_{s}$-metric space, but first we introduce the following notations which are useful in the sequel:

(1) $\mathrm{m}_{\mathrm{s}_{\mathrm{x}, y, z}}=\min \left\{\mathrm{m}_{\mathrm{s}}(x, x, x), \mathrm{m}_{\mathrm{s}}(y, y, y), \mathrm{m}_{\mathrm{s}}(z, z, z)\right\}$;

(2) $M_{s_{x, y}, z}=\max \left\{m_{s}(x, x, x), m_{s}(y, y, y), m_{s}(z, z, z)\right\}$.

Definition 1.7 ([8]). An $M_{s}$-metric on a nonempty set $X$ is a function $m_{s}: X^{3} \mapsto \mathbb{R}^{+}$that satisfies the following conditions: for all $x, y, z, t \in X$,

$\left(m_{s} 1\right) m_{s}(x, x, x)=m_{s}(y, y, y)=m_{s}(z, z, z)=m_{s}(x, y, z)$ if and only if $x=y=z$;

$\left(m_{s} 2\right) m_{s_{x, y, z}} \leqslant m_{s}(x, y, z)$;

$\left(m_{s} 3\right) m_{s}(x, x, y)=m_{s}(y, y, x)$;

$\left(m_{s} 4\right)\left(m_{s}(x, y, z)-m_{s_{x, y}, z}\right) \leqslant\left(m_{s}(x, x, t)-m_{s_{x, x} t}\right)+\left(m_{s}(y, y, t)-m_{s_{y, y, t}}\right)+\left(m_{s}(z, z, t)-m_{s_{z, z, t}}\right)$.

Then the pair $\left(X, m_{s}\right)$ is called an $M_{s}$-metric space.

Example 1.8. Let $X=[0, \infty)$ and $m_{s}: X^{3} \mapsto \mathbb{R}^{+}$be a mapping defined by

$$
m_{s}(x, y, z)=\max \{x, y, z\}-\min \{x, y, z\},
$$

for all $x, y, z \in X$. Then $m_{s}$ is an $M_{s}$-metric on $X$.

Example 1.9. Let $X$ be a nonempty set and $d$ be the ordinary metric on $X$. Define the mapping $m_{s}: X^{3} \mapsto$ $[0, \infty)$ by

$$
m_{s}(x, y, z)=d(x, y)+d(x, z)+d(y, z)
$$

for all $x, y, z \in X$. Then $m_{s}$ is an $M_{s}$-metric on $X$.

Example 1.10. Let $X=\{1,2,3\}$ and define a mapping $m_{s}$ on $X$ by

$$
\begin{array}{ll}
m_{s}(1,2,3)=6, & m_{s}(1,1,2)=m_{s}(2,2,1)=10, \quad m_{s}(1,1,3)=m_{s}(3,3,1)=m_{s}(2,2,3)=m_{s}(3,3,2)=7, \\
m_{s}(2,2,2)=9, & m_{s}(3,3,3)=5, \quad m_{s}(1,1,1)=8 .
\end{array}
$$

Then $m_{s}$ is an $M_{s}$-metric on $X$, but $m_{s}$ is not a partial $S$-metric since $m_{s}(1,1,1) \not \leq m_{s}(1,2,3)$. 
Example 1.11. Let $(X, s)$ be an $S$-metric space with the $S$-metric s. Let $\phi:[0, \infty) \mapsto[0, \infty)$ be an injective and nondecreasing mapping such that

$$
\phi(x+y+z) \leqslant \phi(x)+\phi(y)+\phi(z)-2 \phi(0) .
$$

Then $\mathrm{m}_{\mathrm{s}}(x, y, z)=\phi(s(x, y, z))$ is an $M_{s}$-metric on $X$.

Proof. It is easy to prove that the conditions $\left(m_{s} 1\right),\left(m_{s} 2\right)$, and $\left(m_{s} 3\right)$ are satisfied. For the condition $\left(m_{s} 4\right)$, it follows that, for for all $x, y, z, t \in X$,

$$
\begin{aligned}
\phi(s(x, y, z)) & \leqslant \phi(s(x, x, t)+s(y, y, t)+s(z, z, t)) \\
& \leqslant \phi(s(x, x, t))+\phi(s(y, y, t))+\phi(s(z, z, t))-2 \phi(0) \\
\phi(s(x, y, z))-\phi(0) & \leqslant(\phi(s(x, x, t))-\phi(0))+(\phi(s(y, y, t))-\phi(0))+(\phi(s(z, z, t))-\phi(0)), \\
\left(m_{s}(x, y, z)-m_{s_{x, y, z}}\right) & \leqslant\left(m_{s}(x, x, t)-m_{s_{x, x}, t}\right)+\left(m_{s}(y, y, t)-m_{s_{y, y, t}}\right)+\left(m_{s}(z, z, t)-m_{s_{z, z, t}}\right) .
\end{aligned}
$$

This completes the proof.

Example 1.12. Let $(X, s)$ be an $S$-metric space with the $S$-metric $s$. Then the mapping $m_{s}$ defined by $m_{s}(x, y, z)=a s(x, y, z)+b$ for all $x, y, z \in X$, where $a, b>0$, is an $M_{s}$-metric since we can put $\phi(t)=$ $a t+b$.

Remark 1.13. According to Example 1.12, from Banach's contraction, that is, there exists $k \in[0,1)$ such that

$$
m_{s}(T x, T y, T z) \leqslant k m_{s}(x, y, z)
$$

for all $x, y, z \in X$, we have

$$
m_{s}(T x, T y, T z)=a s(T x, T y, T z)+b \leqslant k a s(x, y, z)+k b \Rightarrow s(T x, T y, T z) \leqslant k s(x, y, z)+\frac{b(k-1)}{a},
$$

which does not imply the ordinary Banach contraction in S-metric spaces (see [11]), that is, there exists $k \in[0,1)$ such that

$$
s(T x, T x, T y) \leqslant k s(x, x, y)
$$

for all $x, y \in X$, where $T$ is a self-mapping on $X$.

Conversely, the ordinary Banach contraction in S-metric spaces implies Banach's contraction in $M_{s^{-}}$ metric spaces. Thus this states that, even if the $M_{s}$-metric $m_{s}$ and the ordinary $S$-metric $s$ have the same topology, Banach's contraction for the $M_{s}$-metric $m_{s}$ does not imply Banach's contraction for the ordinary S-metric s.

For example, let $X$ be a nonempty set and $d$ be the ordinary metric on $X$. Define two mappings $s: X^{3} \mapsto[0, \infty)$ and $m_{s}: X^{3} \mapsto[0, \infty)$ by

$$
s(x, y, z)=d(x, z)+d(y, z), \quad m_{s}(x, y, z)=d(x, y)+d(x, z)+d(y, z)
$$

for all $x, y, z \in X$, respectively. Then $s$ and $m_{s}$ are an $S$-metric and an $M_{s}$-metric, respectively. Obviously, it follows that the $S$-metric $s$ and the $M_{s}$-metric $m_{s}$ generate two topologies $\tau_{s}$ and $\tau_{m_{s}}$ on $X$, respectively, where

$$
B_{s}(x, \epsilon):=\{y \in X: s(x, x, y)<\epsilon\}=\{y \in X: d(x, y)<\epsilon\}
$$

and

$$
B_{m_{s}}(x, \epsilon):=\left\{y \in X: m_{s}(x, x, y)-m_{s_{x, x} y}<\epsilon\right\}=\{y \in X: 2 d(x, y)<\epsilon\}
$$


form the base of $\tau_{s}$ and $\tau_{m_{s}}$, respectively. Notice that $\tau_{s}$ and $\tau_{m_{s}}$ are the same topology. However, by Banach's contraction in $M_{s}$-metric spaces, we have

$$
m_{s}(T x, T x, T y)<k m_{s}(x, x, y) \Rightarrow d(T x, T y)<2 k d(x, y),
$$

for some $k \in[0,1)$, which does not imply the ordinary Banach contraction in S-metric, that is,

$$
s(T x, T x, T y)<k s(x, x, y) \Rightarrow d(T x, T y)<k d(x, y),
$$

for some $k \in[0,1)$.

Lemma 1.14. Let $\left(X, m_{s}\right)$ be an $M_{s}$-metric space. Then, for all $x, y, z, t \in X$,

(1) $m_{s}(x, y, y) \leqslant m_{s}(x, x, y)$;

(2) $m_{s}(x, y, x) \leqslant 2 m_{s}(x, x, y)$;

(3) $m_{s}(x, y, z)-m_{s_{x, y, z}} \leqslant\left(m_{s}(x, x, z)-m_{s_{x, x}, z}\right)+\left(m_{s}(y, y, z)-m_{s_{y, y, z}}\right)$;

(4) $m_{s}(x, y, z)-m_{s_{x, y, z}} \leqslant\left(m_{s}(x, x, y)-m_{s_{x, x}, y}\right)+\left(m_{s}(z, z, y)-m_{s_{z, z, y}}\right)$;

(5) $m_{s}(x, y, z)-m_{s_{x, y, z}} \leqslant\left(m_{s}(y, y, x)-m_{s_{y, y, x}}\right)+\left(m_{s}(z, z, x)-m_{s_{z, z, x}}\right)$;

(6) $m_{s}(x, y, z)-m_{s_{x, y, z}} \leqslant \frac{2}{3}\left[\left(m_{s}(x, x, z)-m_{s_{x, x, z}}\right)+\left(m_{s}(z, z, y)-m_{s_{z, z, y}}\right)+\left(m_{s}(y, y, x)-m_{s_{y, y, x}}\right)\right]$;

(7) $\left(M_{s_{x, y}, z}-m_{s_{x, y}, z}\right) \leqslant\left(M_{s_{x, x, t}}-m_{s_{x, x, t}}\right)+\left(M_{s_{y, y, t}}-m_{s_{y, y, t}}\right)+\left(M_{s_{z, z, t}}-m_{s_{z, z, t}}\right)$.

Proof. (1)-(7) can be directly obtained from Definition 1.7.

Remark 1.15. If $m_{s}$ is an $M_{s}$-metric on a nonempty set $X$, then two mappings $m_{s}^{w}, m_{s}^{*}: X^{2} \mapsto \mathbb{R}^{+}$defined by

$$
m_{s}^{w}(x, y, z)=m_{s}(x, y, z)-2 m_{s_{x, y, z}}+M_{s_{x, y}, z}
$$

and

$$
m_{s}^{*}(x, y, z)= \begin{cases}m_{s}(x, y, z)-m_{s_{x, y}, z}, & x \neq y \neq z, \\ 0, & x=y=z=0,\end{cases}
$$

for all $x, y, z \in X$ are two ordinary S-metrics on $X$. In fact, if $m_{s}^{w}(x, y, z)=0$, then we have

$$
m_{s}(x, y, z)=2 m_{s_{x, y}, z}-M_{s_{x, y}, z} .
$$

But, from equation (1.1) and $\left(m_{s} 2\right)$, it follows that

$$
m_{s_{x, y}, z}=M_{s_{x, y}, z}=m_{s}(x, x, x)=m_{s}(y, y, y)=m_{s}(z, z, z) .
$$

So, by equation (1.1), we have that $m_{s}(x, y, z)=m_{s}(x, x, x)=m_{s}(y, y, y)=m_{s}(z, z, z)$ and so $x=y=z$. We can get the inequality property in the definition of an S-metric from Lemma $1.14(7)$ and $\left(m_{s} 4\right)$.

\section{Topologies for $M_{s}$-metrics}

It is clear that each $M_{s}$-metric $m_{s}$ on $X$ generates a topology $\tau_{m_{s}}$ on $X$. The set

$$
\left\{\mathrm{B}_{\mathrm{m}_{\mathrm{s}}}(x, \epsilon): x \in X, \epsilon>0\right\},
$$

where

$$
B_{m_{s}}(x, \epsilon):=\left\{y \in X: m_{s}(x, x, y)-m_{s_{x, x}, y}<\epsilon\right\},
$$

for all $x \in X$ and $\epsilon>0$, forms a base of $\tau_{m_{s}}$. 
Definition 2.1. Let $\left(X, m_{s}\right)$ be an $M_{s}$-metric space. Then:

(1) A sequence $\left\{x_{n}\right\}$ in $X$ is said to be convergent to a point $x$ if

$$
\lim _{n \rightarrow \infty}\left(m_{s}\left(x_{n}, x_{n}, x\right)-m_{s_{x_{n}, x_{n}, x}}\right)=0 .
$$

(2) A sequence $\left\{x_{n}\right\}$ in $X$ is called an $M_{s}$-Cauchy sequence if $\lim _{n, m \rightarrow \infty}\left(m_{s}\left(x_{n}, x_{n}, x_{m}\right)-m_{s_{x_{n}, x_{n}, x_{m}}}\right)$ and $\lim _{n, m \rightarrow \infty}\left(M_{s_{x_{n}, x_{n}, x_{m}}}-m_{s_{x_{n}, x_{n}, x_{m}}}\right)$ exist and finite.

(3) An $M_{s}$-metric space is said to be complete if every $m_{s}$-Cauchy sequence $\left\{x_{n}\right\}$ in $X$ converges, with respect to $\tau_{m_{s}}$, to a point $x \in X$ such that

$$
\lim _{n \rightarrow \infty}\left(m_{s}\left(x_{n}, x_{n}, x\right)-m_{s_{x_{n}, x_{n}, x}}\right)=0, \quad \lim _{n \rightarrow \infty}\left(M_{s_{x_{n}, x_{n}, x}}-m_{s_{x_{n}, x_{n}, x}}\right)=0 .
$$

Lemma 2.2. Let $\left(\mathrm{X}, \mathrm{m}_{\mathrm{s}}\right)$ be an $\mathrm{M}_{\mathrm{s}}$-metric space. Then:

(1) $\left\{x_{n}\right\}$ is an $M_{s}$-Cauchy sequence in $\left(X, m_{s}\right)$ if and only if it is an S-Cauchy sequence in the S-metric space $\left(\mathrm{X}, \mathrm{m}_{\mathrm{s}}^{\mathrm{w}}\right)$.

(2) An $\mathrm{M}_{\mathrm{s}}$-metric space $\left(\mathrm{X}, \mathrm{m}_{\mathrm{s}}\right)$ is complete if and only if the S-metric space $\left(\mathrm{X}, \mathrm{m}_{\mathrm{s}}^{w}\right)$ is complete. Furthermore,

$$
\lim _{n \rightarrow \infty} m_{s}^{w}\left(x_{n}, x_{n}, x\right)=0 \Longrightarrow \lim _{n \rightarrow \infty}\left(m_{s}\left(x_{n}, x_{n}, x\right)-m_{s_{x_{n}, x_{n}, x}}\right)=0, \lim _{n \rightarrow \infty}\left(M_{s_{x_{n}, x_{n}, x}}-m_{s_{x_{n}, x_{n}, x}}\right)=0 .
$$

Proof. It obviously follows from the definitions of $M_{s}$-Cauchy sequence, $M_{s}$-completeness, S-Cauchy sequence and S-completeness.

Meanwhile, the above assertions are true for $m_{s}^{*}$.

Lemma 2.3. Assume that $\mathrm{x}_{\mathrm{n}} \rightarrow \mathrm{x}$ and $\mathrm{y}_{\mathrm{n}} \rightarrow \mathrm{y}$ as $\mathrm{n} \rightarrow \infty$ in an $\mathrm{M}_{\mathrm{s}}$-metric space $\left(\mathrm{X}, \mathrm{m}_{\mathrm{s}}\right)$. Then

$$
\lim _{n \rightarrow \infty} m_{s}\left(x_{n}, x_{n}, y_{n}\right)-m_{s_{x_{n}, x_{n}, y n}}=m_{s}(x, x, y)-m_{s_{x, x}, y} \text {. }
$$

Proof. We have

$$
\begin{aligned}
& \left|\left(m_{s}\left(x_{n}, x_{n}, y_{n}\right)-m_{s_{x_{n}, x_{n}, y_{n}}}\right)-\left(m_{s}(x, x, y)-m_{s_{x, x}, y}\right)\right| \\
& \quad \leqslant 2\left|m_{s}\left(x_{n}, x_{n}, x\right)-m_{s_{x_{n}, x_{n}, x}}\right|+2\left|m_{s}\left(y_{n}, y_{n}, y\right)-m_{s_{y_{n}, y_{n}, y}}\right| .
\end{aligned}
$$

From Lemma 2.3, we can deduce the following lemma.

Lemma 2.4. Assume that $\mathrm{x}_{\mathrm{n}} \rightarrow \mathrm{x}$ as $\mathrm{n} \rightarrow \infty$ in an $\mathrm{M}_{\mathrm{s}}$-metric space $\left(\mathrm{X}, \mathrm{m}_{\mathrm{s}}\right)$. Then

$$
\lim _{n \rightarrow \infty} m_{s}\left(x_{n}, x_{n}, y\right)-m_{s_{x_{n}, x_{n}, y}}=m_{s}(x, x, y)-m_{s_{x, x}, y}
$$

for all $\mathrm{y} \in \mathrm{X}$.

Lemma 2.5. Assume that $x_{n} \rightarrow x$ and $x_{n} \rightarrow y$ as $n \rightarrow \infty$ in an $M_{s}$-metric space $\left(X, m_{s}\right)$. Then, $m_{s}(x, x, y)=$ $m_{s_{x, x}, y}$. Furthermore, if $\mathrm{m}_{\mathrm{s}}(x, x, x)=\mathrm{m}_{\mathrm{s}}(\mathrm{y}, \mathrm{y}, \mathrm{y})$, then $\mathrm{x}=\mathrm{y}$.

Proof. By Lemma 2.3, we have

$$
0=\lim _{n \rightarrow \infty} m_{s}\left(x_{n}, x_{n}, x_{n}\right)-m_{s_{x_{n}, x_{n}, x_{n}}}=m_{s}(x, x, y)-m_{s_{x, x}, y} .
$$

Lemma 2.6. Let $\left\{x_{n}\right\}$ be a sequence in $M_{s}$-metric space $\left(X, m_{s}\right)$ such that there exists $r \in[0,1)$ such that

$$
m_{s}\left(x_{n+1}, x_{n+1}, x_{n}\right) \leqslant r m_{s}\left(x_{n}, x_{n}, x_{n-1}\right),
$$

for all $\mathrm{n} \in \mathbb{N}$. Then we have

(1) $\lim _{n \rightarrow \infty} m_{s}\left(x_{n}, x_{n}, x_{n-1}\right)=0$;

(2) $\lim _{n \rightarrow \infty} m_{s}\left(x_{n}, x_{n}, x_{n}\right)=0$; 
(3) $\lim _{n, m \rightarrow \infty} m_{s_{x_{n}, x_{n}, x_{m}}}=0$;

(4) $\left\{x_{n}\right\}$ is an $M_{s}-$ Cauchy sequence.

Proof. From the equation (2.1), we have

$$
m_{s}\left(x_{n}, x_{n}, x_{n-1}\right) \leqslant r m_{s}\left(x_{n-1}, x_{n-1}, x_{n-2}\right) \leqslant r^{2} m_{s}\left(x_{n-2}, x_{n-2}, x_{n-3}\right) \leqslant \cdots \leqslant r^{n} m_{s}\left(x_{1}, x_{1}, x_{0}\right)
$$

and so $\lim _{n \rightarrow \infty} m_{s}\left(x_{n}, x_{n}, x_{n-1}\right)=0$, which implies that (A) holds. From $\left(m_{s} 2\right)$ and (1), we have

$$
\lim _{n \rightarrow \infty} m_{s_{x_{n}, x_{n}, x_{n-1}}} \leqslant \lim _{n \rightarrow \infty} m_{s}\left(x_{n}, x_{n}, x_{n-1}\right)=0,
$$

that is, (2) holds. Clearly, (3) and (4) hold.

Theorem 2.7. The topology $\tau_{\mathrm{m}_{\mathrm{s}}}$ is not Hausdorff.

Proof. Let $x, y, z \in X$ be such that $a:=m_{s}(x, x, x)<m_{s}(z, z, z)=\frac{a+b}{2}<b:=m_{s}(y, y, y)$ with

$$
\begin{aligned}
& \frac{b}{2}<\frac{k}{2}<m_{s}(y, y, y)<M_{s_{x, x}, y}=b, r=2 m_{s}(x, x, y)-a-b>0, \\
& \max \left\{m_{s}(x, x, z), m_{s}(z, z, y)\right\} \leqslant\left(2 m_{s}(x, x, y)-k\right) \frac{\epsilon}{r} .
\end{aligned}
$$

Without loss of generality, we assume that, for each $\epsilon>0, \epsilon<r$. Now, we need to prove that the intersection of the following neighborhoods is not empty:

$$
\mathrm{U}_{x}=\left\{z \in X: \mathrm{m}_{\mathrm{s}}(x, x, z)-\mathrm{m}_{s_{x, x, z}}<\epsilon\right\}, \quad \mathrm{V}_{y}=\left\{z \in X: \mathrm{m}_{s}(y, y, z)-\mathrm{m}_{s_{y, y, z}}<\epsilon\right\} .
$$

To prove $z \in \mathrm{U}_{x}$, we have

$$
\begin{aligned}
m_{s}(x, x, z) & <\left(2 m_{s}(x, x, y)-k\right) \frac{\epsilon}{r}, \\
m_{s}(x, x, z)-m_{s_{x, x}, z} & <\left(2 m_{s}(x, x, y)-k\right) \frac{\epsilon}{r}-a<\left(2 m_{s}(x, x, y)-k-a\right) \frac{\epsilon}{r}<\left(2 m_{s}(x, x, y)-a-b\right) \frac{\epsilon}{r}=\epsilon
\end{aligned}
$$

and, for any $z \in V_{y}$, we also have

$$
\begin{aligned}
m_{s}(y, y, z) & <\left(2 m_{s}(x, x, y)-k\right) \frac{\epsilon}{r} \\
m_{s}(y, y, z)-m_{s_{y, y, z}} & <\left(2 m_{s}(x, x, y)-k\right) \frac{\epsilon}{r}-\frac{a+b}{2} \\
& <\left(2 m_{s}(x, x, y)-k\right) \frac{\epsilon}{r}-\frac{a+b}{2} \frac{\epsilon}{r} \\
& <\left(2 m_{s}(x, x, y)-k-\frac{a+b}{2}\right) \frac{\epsilon}{r}<\left(2 m_{s}(x, x, y)-a-b\right) \frac{\epsilon}{r}=\epsilon .
\end{aligned}
$$

So, we can find $x, y \in X$ such that, for all nonempty neighborhoods $u_{x}$ of $x$ and $v_{y}$ of $y, u_{x} \cap v_{y} \neq \emptyset$. This completes the proof.

\section{Main results}

3.1. On fixed point problems under Banach's contractive condition in $\mathrm{M}_{\mathrm{s}}$-metric spaces

In this section, we introduce the concepts of the Ulam-Hyers stability, the well-posedness and the limit shadowing property of the fixed point problem in $M_{s}$-metric spaces. Then we study the corresponding results for the fixed point problem under Banach's contractive condition in $M_{s}$-metric spaces. 
Definition 3.1. Let $\left(X, m_{s}\right)$ be an $M_{s}$-metric space and $T: X \mapsto X$ be a mapping. The fixed point problem

$$
x=\mathrm{T} x
$$

is said to be Ulam-Hyers stable if there exists $c>0$ such that, for any $\epsilon>0$ and for each $w^{*} \in X$ which is an $\epsilon$-solution of the fixed point problem (3.1), i.e., $w^{*}$ satisfies the inequality

$$
\mathrm{m}_{\mathrm{s}}\left(w^{*}, w^{*}, T w^{*}\right) \leqslant \epsilon,
$$

there exists a solution $x^{*} \in X$ of the equation (3.1) such that

$$
\mathrm{m}_{\mathrm{s}}\left(x^{*}, w^{*}, w^{*}\right) \leqslant c \epsilon .
$$

Definition 3.2. Let $\left(X, m_{s}\right)$ be an $M_{s}$-metric space and $T: X \mapsto X$ be a mapping. The fixed point problem of $\mathrm{T}$ is said to be well-posed if the following conditions hold:

(1) $T$ has a unique fixed point $x^{*}$ of $T$;

(2) for any sequence $\left\{x_{n}\right\}$ in $X$ with $\lim _{n \rightarrow \infty} m_{s}\left(x_{n}, T x_{n}, T x_{n}\right)=0$, we have $\lim _{n \rightarrow \infty} m_{s}\left(x^{*}, x_{n}, x_{n}\right)=0$.

Definition 3.3. Let $\left(X, m_{s}\right)$ be an $M_{s}$-metric space and $T: X \mapsto X$ be a mapping. The fixed point problem of $T$ is said to have the limit shadowing property in $X$ if, for any sequence $\left\{x_{n}\right\}$ in $X$ with $\lim _{n \rightarrow \infty} m_{s}\left(x_{n}, T x_{n}, T x_{n}\right)=0$, it follows that there exists $z \in X$ such that $\lim _{n \rightarrow \infty} m_{s}\left(T^{n} z, x_{n}, x_{n}\right)=0$.

Theorem 3.4. Let $\left(X, \mathrm{~m}_{\mathrm{s}}\right)$ be an $\mathrm{M}_{\mathrm{s}}$-metric space and $\mathrm{T}: \mathrm{X} \mapsto \mathrm{X}$ be a Banach contractive mapping with constant $k \in[0,1)$. Then the following assertions hold:

(1) the fixed point problem of $\mathrm{T}$ is Ulam-Hyers stable;

(2) the fixed point problem of $\mathrm{T}$ is well-posed;

(3) the fixed point problem of $\mathrm{T}$ has the limit shadowing property in $\mathrm{X}$.

Proof. From Theorem 1.3, it follows that $\mathrm{T}$ has a unique fixed point $x^{*} \in \mathrm{X}$ with $\mathrm{m}_{\mathrm{s}}\left(\mathrm{x}^{*}, \mathrm{x}^{*}, \mathrm{x}^{*}\right)=0$.

Next, we first claim that the fixed point problem of T is Ulam-Hyers stable. Let $\frac{\epsilon}{2}>0$ and $w^{*} \in X$ be an $\frac{\epsilon}{2}$-solution of (3.1), i.e.,

$$
m_{s}\left(w^{*}, w^{*}, T w^{*}\right) \leqslant \frac{\epsilon}{2}
$$

From $\left(m_{s} 4\right)$ together with (3.2), we obtain

$$
\begin{aligned}
m_{s}\left(x^{*}, w^{*}, w^{*}\right) \leqslant & {\left[m_{s}\left(x^{*}, x^{*}, T w^{*}\right)-m_{s_{x^{*}, x^{*}, T w^{*}}}\right]+2\left[m_{s}\left(w^{*}, w^{*}, T w^{*}\right)-m_{s_{w^{*}, w^{*}, T w^{*}}}\right]+m_{s_{x^{*}, w^{*}, w^{*}}} } \\
= & {\left[m_{s}\left(T x^{*}, T x^{*}, T w^{*}\right)-m_{s_{x^{*}, x^{*}, T w^{*}}}\right]+2\left[m_{s}\left(w^{*}, w^{*}, T w^{*}\right)-m_{s_{w^{*}, w^{*}, T w^{*}}}\right]+m_{s_{x^{*}, w^{*}, w^{*}}} } \\
\leqslant & {\left[k m_{s}\left(x^{*}, x^{*}, w^{*}\right)-m_{s_{x^{*}, x^{*}, T w^{*}}}\right]+2\left[m_{s}\left(w^{*}, w^{*}, T w^{*}\right)-m_{s_{w^{*}, w^{*}, T w^{*}}}\right]+m_{s_{x^{*}, w^{*}, w^{*}}} } \\
= & {\left[k m_{s}\left(x^{*}, x^{*}, w^{*}\right)-m_{s_{x^{*}, x^{*}, T w^{*}}}\right]+2 m_{s}\left(w^{*}, w^{*}, T w^{*}\right)+\min \left\{m_{s}\left(x^{*}, x^{*}, x^{*}\right), m_{s}\left(w^{*}, w^{*}, w^{*}\right)\right\} } \\
& -2 \min \left\{m_{s}\left(w^{*}, w^{*}, w^{*}\right), m_{s}\left(T w^{*}, T w^{*}, T w^{*}\right)\right\} \\
= & {\left[k m_{s}\left(x^{*}, x^{*}, w^{*}\right)-m_{s_{x^{*}, x^{*}, T w^{*}}}\right]+2 m_{s}\left(w^{*}, w^{*}, T w^{*}\right)-2 m_{s}\left(T w^{*}, T w^{*}, T w^{*}\right) } \\
\leqslant & k m_{s}\left(x^{*}, x^{*}, w^{*}\right)+\epsilon,
\end{aligned}
$$

which implies $m_{s}\left(x^{*}, x^{*}, w^{*}\right) \leqslant c \epsilon$, where $c=\frac{1}{1-k}$. Therefore, the fixed point of $\mathrm{T}$ is Ulam-Hyers stable.

Next, we prove that the fixed point problem of $T$ is well-posed. Assume that $\left\{x_{n}\right\}$ is a sequence in $X$ such that $\lim _{n \rightarrow \infty} m_{s}\left(x_{n}, T x_{n}, T x_{n}\right)=0$. Now, we show that $\lim _{n \rightarrow \infty} m_{s}\left(x^{*}, x_{n}, x_{n}\right)=0$. By $\left(m_{s} 4\right)$, we have

$$
\begin{aligned}
m_{s}\left(x^{*}, x_{n}, x_{n}\right) \leqslant & {\left[m_{s}\left(x^{*}, x^{*}, T x_{n}\right)-m_{s_{x^{*}, x^{*}, T x_{n}}}\right]+2\left[m_{s}\left(x_{n}, x_{n}, T x_{n}\right)-m_{s_{x_{n}, x_{n}, T x_{n}}}\right]+m_{s_{x^{*}, x_{n}, x_{n}}} } \\
= & {\left[m_{s}\left(T x^{*}, T x^{*}, T x_{n}\right)-m_{s_{x^{*}, x^{*}, T x_{n}}}\right]+2\left[m_{s}\left(x_{n}, x_{n}, T x_{n}\right)-m_{s_{x_{n}, x_{n}, T x_{n}}}\right]+m_{s_{x^{*}, x_{n}, x_{n}}} } \\
\leqslant & {\left[k m_{s}\left(x^{*}, x^{*}, x_{n}\right)-m_{s_{x^{*}, x^{*}, T x_{n}}}\right]+2 m_{s}\left(x_{n}, x_{n}, T x_{n}\right)+m_{s_{x^{*}, x_{n}, x_{n}}}-2 m_{s_{x_{n}, x_{n}, T x_{n}}} } \\
= & {\left[k m_{s}\left(x^{*}, x^{*}, x_{n}\right)-m_{s_{x^{*}, x^{*}, T x_{n}}}\right]+2 m_{s}\left(x_{n}, x_{n}, T x_{n}\right) } \\
& -2 \min \left\{m_{s}\left(x_{n}, x_{n}, x_{n}\right), m_{s}\left(T x_{n}, T x_{n}, T x_{n}\right)\right\} \\
\leqslant & k m_{s}\left(x^{*}, x^{*}, x_{n}\right)+2 m_{s}\left(x_{n}, x_{n}, T x_{n}\right),
\end{aligned}
$$


for all $n \in \mathbb{N}$, which implies that

$$
m_{s}\left(x^{*}, x^{*}, x_{n}\right) \leqslant \frac{2}{1-k} m_{s}\left(x_{n}, x_{n}, T x_{n}\right),
$$

for all $n \in \mathbb{N}$. Taking the limit as $n \rightarrow \infty$ in (3.3), we have $\lim _{n \rightarrow \infty} m_{s}\left(x^{*}, x^{*}, x_{n}\right)=0$ and hence the fixed point problem of $\mathrm{T}$ is well-posed.

Finally, we prove that $T$ has the limit shadowing property. Let $\left\{x_{n}\right\}$ be any sequence in $X$ such that $\lim _{n \rightarrow \infty} m_{s}\left(x_{n}, T x_{n}, T x_{n}\right)=0$. From the discussion above, we have $\lim _{n \rightarrow \infty} m_{s}\left(x^{*}, x_{n}, x_{n}\right)=0$. Since $x^{*}$ is a fixed point of $T$, we have

$$
\lim _{n \rightarrow \infty} m_{s}\left(x_{n}, x_{n}, T^{n} \chi^{*}\right)=\lim _{n \rightarrow \infty} m_{s}\left(x_{n}, x_{n}, x^{*}\right)=0 .
$$

Therefore, $\mathrm{T}$ has the limit shadowing property. This completes the proof.

Now, we give an example to illustrate the validity of Theorem 3.4.

Example 3.5. Let $X=[0, \infty)$ and $m_{s}: X^{3} \mapsto[0, \infty)$ be a mapping defined by

$$
m_{s}(x, y, z)=\max \{x, y, z\}-\min \{x, y, z\},
$$

for all $x, y, z \in X$. Then $\left(X, m_{s}\right)$ is a complete $M_{s}$-metric space. Define a mapping $T: X \mapsto X$ by $T x=\frac{x}{2}$ for all $x \in X$. For each $x, y, z \in X$, we obtain

$$
m_{s}(T x, T y, T z)=\frac{1}{2}(\max \{x, y, z\}-\min \{x, y, z\})=\frac{1}{2} m_{s}(x, y, z) .
$$

It follows that $T$ is the Banach contraction with constant $k=\frac{1}{2} \in[0,1)$.

First, we claim that the fixed point problem of $T$ is Ulam-Hyers stable. Assume that $\epsilon>0$ and $w^{*} \in X$ is an $\epsilon$-solution of the fixed point problem of $\mathrm{T}$, that is,

$$
\mathrm{m}_{\mathrm{s}}\left(w^{*}, w^{*}, \mathrm{~T} w^{*}\right) \leqslant \epsilon \Longrightarrow \frac{1}{2}\left(\max \left\{w^{*}, w^{*}, \frac{w^{*}}{2}\right\}-\min \left\{w^{*}, w^{*}, \frac{w^{*}}{2}\right\}\right)=\frac{w^{*}}{4} \leqslant \epsilon .
$$

It is easy to see that $x^{*}=0$ is a solution of the fixed point problem of $T$ and

$$
\mathrm{m}_{\mathrm{s}}\left(x^{*}, x^{*}, w^{*}\right)=\frac{1}{2}\left(\max \left\{0,0, w^{*}\right\}-\min \left\{0,0, w^{*}\right\}\right)=\frac{w^{*}}{2} \leqslant 2 \epsilon
$$

with the constant $c$ in Definition 3.1 taken by $2>0$. Hence the fixed point problem of $\mathrm{T}$ is Ulam-Hyers stable.

Next, we prove that the fixed point problem of $T$ is well-posed. We can see that $x^{*}=0$ is a unique fixed point of $T$. Now, we assume that $\left\{x_{n}\right\}$ is a sequence in $X$ such that $\lim _{n \rightarrow \infty} m_{s}\left(x_{n}, T x_{n}, T x_{n}\right)=0$, that is,

$$
\lim _{n \rightarrow \infty} \frac{1}{2}\left(\max \left\{x_{n}, \frac{x_{n}}{2}, \frac{x_{n}}{2}\right\}-\min \left\{x_{n}, \frac{x_{n}}{2}, \frac{x_{n}}{2}\right\}\right)=0 \Longrightarrow \lim _{n \rightarrow \infty} x_{n}=0 .
$$

Then we obtain that $\lim _{n \rightarrow \infty} m_{s}\left(x^{*}, x_{n}, x_{n}\right)=\lim _{n \rightarrow \infty} m_{s}\left(0, x_{n}, x_{n}\right)=\lim _{n \rightarrow \infty} x_{n}=0$ and so the fixed point problem of $\mathrm{T}$ is well-posed.

Finally, we state that the fixed point problem of $T$ has the limit shadowing property in $X$. Suppose that $\left\{x_{n}\right\}$ is any sequence in $X$ such that $\lim _{n \rightarrow \infty} m_{s}\left(x_{n}, T x_{n}, T x_{n}\right)=0$. It follows that $\lim _{n \rightarrow \infty} x_{n}=0$. We can see that there exists $z=0 \in X$ such that

$$
\lim _{n \rightarrow \infty} m_{s}\left(T^{n} z, x_{n}, x_{n}\right)=\lim _{n \rightarrow \infty} m_{s}\left(0, x_{n}, x_{n}\right)=\lim _{n \rightarrow \infty} x_{n}=0,
$$

which implies that the fixed point problem of T has the limit shadowing property in $X$. 


\subsection{On fixed point problems under Kannan's contractive condition in $\mathrm{M}_{\mathrm{s}}$-metric spaces}

The purpose of this section is to introduce another type of the Ulam-Hyers stability, the well-posedness and the limit shadowing property of the fixed point problem in $M_{s}$-metric spaces. Then we also give the analogous results for the fixed point problem of Kannan contractive mappings in $M_{s}$-metric spaces.

Definition 3.6. Let $\left(X, m_{s}\right)$ be an $M_{s}$-metric space and $T: X \mapsto X$ be a mapping. The fixed point problem (3.1) is said to be Ulam-Hyers stable type (K) if there exists $c>0$ such that, for any $\epsilon>0$ and for each $w^{*} \in \mathrm{X}$ which is an $\epsilon$-solution of the fixed point problem (3.1), i.e., $w^{*}$ satisfies the inequality

$$
\mathrm{m}_{\mathrm{s}}\left(w^{*}, w^{*}, T w^{*}\right) \leqslant \epsilon,
$$

there exists a solution $x^{*} \in X$ of the (3.1) such that

$$
\mathrm{m}_{\mathrm{s}}\left(\mathrm{x}^{*}, w^{*}, w^{*}\right)-c \mathrm{~m}_{\mathrm{s}}\left(x^{*}, x^{*}, x^{*}\right) \leqslant c \epsilon .
$$

Remark 3.7. It is obvious that the Ulam-Hyers stability of the fixed problem implies the Ulam-Hyers stability type $(\mathrm{K})$.

Definition 3.8. Let $\left(X, m_{s}\right)$ be an $M_{s}$-metric space and $T: X \mapsto X$ be a mapping. The fixed point problem of $\mathrm{T}$ is said to be well-posed type (K) if the following conditions hold:

(1) Thas a unique fixed point $x^{*}$ of $T$;

(2) there exists $c>0$ such that, for any sequence $\left\{x_{n}\right\}$ in $X$ with $\lim _{n \rightarrow \infty} m_{s}\left(x_{n}, T x_{n}, T x_{n}\right)=0$, we have $\lim _{n \rightarrow \infty} m_{s}\left(x_{n}, x^{*}, x^{*}\right)=c m_{s}\left(x^{*}, x^{*}, x^{*}\right)$.

Definition 3.9. Let $\left(X, m_{s}\right)$ be an $M_{s}$-metric space and $T: X \mapsto X$ be a mapping. The fixed point problem of $T$ is said to have the limit shadowing property type $(K)$ in $X$ if there exists $c>0$ such that for any sequence $\left\{x_{n}\right\}$ in $X$ with $\lim _{n \rightarrow \infty} m_{s}\left(x_{n}, T x_{n}, T x_{n}\right)=0$, it follows that there exists $z \in X$ such that $\lim _{n \rightarrow \infty} m_{s}\left(T^{n} z, x_{n}, x_{n}\right)=c m_{s}(z, z, z)$.

Theorem 3.10. Let $\left(X, m_{s}\right)$ be an $\mathrm{M}_{\mathrm{s}}$-metric space and $\mathrm{T}: \mathrm{X} \mapsto \mathrm{X}$ be a Kannan contractive mapping with constant $k \in\left[0, \frac{1}{2}\right)$. Then the following assertions hold:

(1) The fixed point problem of $\mathrm{T}$ is Ulam-Hyers stable type (K).

(2) The fixed point problem of $\mathrm{T}$ is well-posed type (K).

(3) The fixed point problem of $\mathrm{T}$ has the limit shadowing property type (K) in X.

Proof. From Theorem 1.4, it follows that T has a unique fixed point $x^{*} \in \mathrm{X}$ with $\mathrm{m}_{\mathrm{s}}\left(\mathrm{x}^{*}, \mathrm{x}^{*}, \mathrm{x}^{*}\right)=0$.

Now, we first claim that the fixed point problem of $T$ is Ulam-Hyers stable type (K). Let $\frac{\epsilon}{2}>0$ and $w^{*} \in X$ be an $\frac{\epsilon}{2}$-solution of the equation (3.1), i.e.,

$$
m_{s}\left(w^{*}, w^{*}, T w^{*}\right) \leqslant \frac{\epsilon}{2} .
$$

From $\left(m_{s} 4\right)$ together with (3.4), we obtain

$$
\begin{aligned}
m_{s}\left(x^{*}, w^{*}, w^{*}\right) & \leqslant\left[m_{s}\left(x^{*}, x^{*}, T w^{*}\right)-m_{s_{x^{*}, x^{*}, T w^{*}}}\right]+2\left[m_{s}\left(w^{*}, w^{*}, T w^{*}\right)-m_{s_{w^{*}, w^{*}, T w^{*}}}\right]+m_{s_{x^{*}, w^{*}, w^{*}}} \\
& =\left[m_{s}\left(T x^{*}, T x^{*}, T w^{*}\right)-m_{s_{x^{*}, x^{*}, T w^{*}}}\right]+2\left[m_{s}\left(w^{*}, w^{*}, T w^{*}\right)-m_{s_{w^{*}, w^{*}, T w^{*}}}\right]+m_{s_{x^{*}, w^{*}, w^{*}}} \\
& \leqslant m_{s}\left(T x^{*}, T x^{*}, T w^{*}\right)+2 m_{s}\left(w^{*}, w^{*}, T w^{*}\right)+m_{s_{x^{*}, w^{*}, w^{*}}} \\
& \leqslant k\left[m_{s}\left(x^{*}, x^{*}, T x^{*}\right)+m_{s}\left(w^{*}, w^{*}, T w^{*}\right)\right]+2 m_{s}\left(w^{*}, w^{*}, T w^{*}\right)+m_{s_{x^{*}, w^{*}, w^{*}}} \\
& =k\left[m_{s}\left(x^{*}, x^{*}, x^{*}\right)+m_{s}\left(w^{*}, w^{*}, T w^{*}\right)\right]+2 m_{s}\left(w^{*}, w^{*}, T w^{*}\right)+m_{s}\left(x^{*}, x^{*}, x^{*}\right) \\
& \leqslant(k+1) m_{s}\left(x^{*}, x^{*}, x^{*}\right)+(2+k) \epsilon \\
& \leqslant(k+2)\left[m_{s}\left(x^{*}, x^{*}, x^{*}\right)+\epsilon\right],
\end{aligned}
$$


which implies $m_{s}\left(x^{*}, x^{*}, w^{*}\right)-c_{s}\left(x^{*}, x^{*}, x^{*}\right) \leqslant c \epsilon$, where $c=k+2>0$. Thus the fixed point of $\mathrm{T}$ is Ulam-Hyers stable $(\mathrm{K})$.

Next, we prove that the fixed point problem of $T$ is well-posed type (K). Assume that $\left\{x_{n}\right\}$ is a sequence in $X$ such that $\lim _{n \rightarrow \infty} m_{s}\left(x_{n}, T x_{n}, T x_{n}\right)=0$. Now, we show that $\lim _{n \rightarrow \infty} m_{s}\left(x^{*}, x_{n}, x_{n}\right)=0$. By $\left(m_{s} 4\right)$, it follows that

$$
\begin{aligned}
m_{s}\left(x^{*}, x_{n}, x_{n}\right) & \leqslant\left[m_{s}\left(x^{*}, x^{*}, T x_{n}\right)-m_{s_{x^{*}, x^{*}, T x_{n}}}\right]+2\left[m_{s}\left(x_{n}, x_{n}, T x_{n}\right)-m_{s_{x_{n}, x_{n}, T x_{n}}}\right]+m_{s_{x^{*}, x_{n}, x_{n}}} \\
& =\left[m_{s}\left(T x^{*}, T x^{*}, T x_{n}\right)-m_{s_{x^{*}, x^{*}, T x_{n}}}\right]+2\left[m_{s}\left(x_{n}, x_{n}, T x_{n}\right)-m_{s_{x_{n}, x_{n}, T x_{n}}}\right]+m_{s_{x^{*}, x_{n}, x_{n}}} \\
& \leqslant m_{s}\left(T x^{*}, T x^{*}, T x_{n}\right)+2 m_{s}\left(x_{n}, x_{n}, T x_{n}\right)+m_{s_{x^{*}, x_{n}, x_{n}}} \\
& \leqslant k\left[m_{s}\left(x^{*}, x^{*}, T x^{*}\right)+m_{s}\left(x_{n}, x_{n}, T x_{n}\right)\right]+2 m_{s}\left(x_{n}, x_{n}, T x_{n}\right)+m_{s_{x^{*}, x_{n}, x_{n}}} \\
& =k\left[m_{s}\left(x^{*}, x^{*}, x^{*}\right)+m_{s}\left(x_{n}, x_{n}, T x_{n}\right)\right]+2 m_{s}\left(x_{n}, x_{n}, T x_{n}\right)+m_{s}\left(x^{*}, x^{*}, x^{*}\right) \\
& \leqslant(k+2)\left[m_{s}\left(x^{*}, x^{*}, x^{*}\right)+m_{s}\left(x_{n}, x_{n}, T x_{n}\right)\right],
\end{aligned}
$$

for all $n \in \mathbb{N}$. Taking the limit as $n \rightarrow \infty$ in the last inequality above, we have

$$
\lim _{n \rightarrow \infty} m_{s}\left(x_{n}, x_{n}, T^{n} x^{*}\right)=\lim _{n \rightarrow \infty} m_{s}\left(x_{n}, x_{n}, x^{*}\right)=(k+2) m_{s}\left(x^{*}, x^{*}, x^{*}\right) .
$$

Hence the fixed point problem of $\mathrm{T}$ is well-posed type $(\mathrm{K})$.

Finally, we prove that $T$ has the limit shadowing property type $(K)$. Let $\left\{x_{n}\right\}$ be any sequence in $X$ such that $\lim _{n \rightarrow \infty} m_{s}\left(x_{n}, T x_{n}, T x_{n}\right)=0$. Since $x^{*}$ is a fixed point of $T$, from (3.5), we have $\lim _{n \rightarrow \infty} m_{s}\left(x^{*}, x_{n}, x_{n}\right)=0$. Since $x^{*}$ is a fixed point of $T$, we have

$$
\lim _{n \rightarrow \infty} m_{s}\left(x_{n}, x_{n}, T^{n} x^{*}\right)=\lim _{n \rightarrow \infty} m_{s}\left(x_{n}, x_{n}, x^{*}\right)=(k+2) m_{s}\left(x^{*}, x^{*}, x^{*}\right) .
$$

Therefore, $\mathrm{T}$ has the limit shadowing property type $(\mathrm{K})$. This completes the proof.

Now, we present an example to illustrate Theorem 3.10.

Example 3.11. Let $X=[0, \infty)$ and define a mapping $m_{s}: X^{3} \mapsto[0, \infty)$ by

$$
m_{s}(x, y, z)=\max \left\{x^{2}, y^{2}, z^{2}\right\}-\min \left\{x^{2}, y^{2}, z^{2}\right\},
$$

for all $x, y, z \in X$. Then $\left(X, m_{s}\right)$ is a complete $M_{s}$-metric space. Define a mapping $T: X \mapsto X$ by $T x=\frac{x}{2}$ for all $x \in X$. For each $x, y \in X$, we obtain

$$
\begin{aligned}
m_{s}(T x, T x, T y) & =\left(\max \left\{\frac{x^{2}}{4}, \frac{x^{2}}{4}, \frac{y^{2}}{4}\right\}-\min \left\{\frac{x^{2}}{4}, \frac{x^{2}}{4}, \frac{y^{2}}{4}\right\}\right) \\
& =\left|\frac{x^{2}}{4}-\frac{y^{2}}{4}\right| \\
& \leqslant\left|\frac{x^{2}}{4}\right|+\left|\frac{y^{2}}{4}\right| \\
& =\frac{1}{3}\left[\left(\max \left\{\frac{x^{2}}{4}, \frac{x^{2}}{4}, x^{2}\right\}-\min \left\{\frac{x^{2}}{4}, \frac{x^{2}}{4}, x^{2}\right\}\right)+\left(\max \left\{\frac{y^{2}}{4}, \frac{y^{2}}{4}, y^{2}\right\}-\min \left\{\frac{y^{2}}{4}, \frac{y^{2}}{4}, y^{2}\right\}\right)\right] \\
& =\frac{1}{3}\left(m_{s}(x, x, T x)+m_{s}(y, y, T y) .\right.
\end{aligned}
$$

It follows that $T$ is a Kannan contractive mapping with constant $k=\frac{1}{3} \in\left[0, \frac{1}{2}\right)$.

First, we claim that the fixed point problem of $\mathrm{T}$ is Ulam-Hyers stable type (K). Assume that $\epsilon>0$ and $w \in \mathrm{X}$ is an $\epsilon$-solution of the fixed point problem of $\mathrm{T}$, that is,

$$
\mathrm{m}_{\mathrm{s}}(w, w, \mathrm{~T} w) \leqslant \epsilon \Longrightarrow\left(\max \left\{w^{2}, w^{2}, \frac{w^{2}}{4}\right\}-\min \left\{w^{2}, w^{2}, \frac{w^{2}}{4}\right\}\right)=\frac{3 w^{2}}{4} \leqslant \epsilon .
$$


It is easy to see that $x^{*}=0$ is a solution of the fixed point problem of $T$ and

$$
\mathrm{m}_{\mathrm{s}}\left(x^{*}, x^{*}, w\right)=\left(\max \left\{0,0, w^{2}\right\}-\min \left\{0,0, w^{2}\right\}\right)=w^{2} \leqslant \frac{4}{3} \epsilon
$$

with the constant $c$ in Definition 3.6 taken by $\frac{4}{3}>0$. Hence the fixed point problem of $\mathrm{T}$ is UlamHyers stable type $(K)$. Now, we prove that the fixed point problem of $T$ is well-posed type $(K)$. We can see that $x^{*}=0$ is a unique fixed point of $T$. We assume that $\left\{x_{n}\right\}$ is a sequence in $X$ such that $\lim _{n \rightarrow \infty} m_{s}\left(x_{n}, T x_{n}, T x_{n}\right)=0$, that is,

$$
\lim _{n \rightarrow \infty}\left(\max \left\{x_{n}^{2}, \frac{x_{n}^{2}}{4}, \frac{x_{n}^{2}}{4}\right\}-\min \left\{x_{n}^{2}, \frac{x_{n}^{2}}{4}, \frac{x_{n}^{2}}{4}\right\}\right)=\lim _{n \rightarrow \infty} \frac{3 x_{n}^{2}}{4}=0 \Longrightarrow \lim _{n \rightarrow \infty} x_{n}^{2}=0
$$

Then we obtain

$$
\lim _{n \rightarrow \infty} m_{s}\left(x^{*}, x_{n}, x_{n}\right)=\lim _{n \rightarrow \infty} m_{s}\left(0, x_{n}, x_{n}\right)=\lim _{n \rightarrow \infty} x_{n}^{2}=0
$$

and so the fixed point problem of $\mathrm{T}$ is well-posed type (K).

Finally, we state that the fixed point problem of $T$ has the limit shadowing property type $(K)$ in $X$. Suppose that $\left\{x_{n}\right\}$ is any sequence in $X$ such that $\lim _{n \rightarrow \infty} m_{s}\left(x_{n}, T x_{n}, T x_{n}\right)=0$. It follows that $\lim _{n \rightarrow \infty} x_{n}^{2}=0$. We can see that there exists $z=0 \in X$ such that

$$
\lim _{n \rightarrow \infty} m_{s}\left(T^{n} z, x_{n}, x_{n}\right)=\lim _{n \rightarrow \infty} m_{s}\left(0, x_{n}, x_{n}\right)=\lim _{n \rightarrow \infty} x_{n}^{2}=0,
$$

which implies that the fixed point problem of $T$ has the limit shadowing property type $(K)$ in $X$.

\section{Acknowledgment}

The first author was supported in part by Natural Science Foundation of Hainan Province (Grant No.114014), Scientific Research Fund of Hainan Province Education Department (Grant No.Hnjg2016ZD20). The second author was partially supported by National Natural Science Foundation of China (Grant No.61573010), Opening Project of Sichuan Province University Key Laboratory of Bridge Nondestruction Detecting and Engineering Computing (No.2015QZJ01), Artificial Intelligence of Key Laboratory of Sichuan Province (No.2015RZJ01), Scientific Research Fund of Sichuan Provincial Education Department (No.16ZA0256), Science Research Fund of Science and Technology Department of Sichuan Province (N0.2017JY0125), Scientific Research Fund of Sichuan University of Science and Engineering (No.2014RC01 No.2014RC03). The forth authors are thankful to the Ministry of Education, Science and Technological Development of Serbia.

\section{References}

[1] S. Banach, Sur les opérations dans les ensembles abstraits et leur application aux équations intégrales, Fund. Math., 3 (1922), 133-181. 1, 1.1

[2] M. F. Bota, E. Karapınar, O. Mleşniţe, Ulam-Hyers stability results for fixed point problems via $\alpha$ - $\psi$-contractive mapping in (b)-metric space, Abstr. Appl. Anal., 2013 (2013), 6 pages. 1

[3] M. F. Bota-Boriceanu, A. Petruşel, Ulam-Hyers stability for operatorial equations, An. Ştiinţ. Univ. Al. I. Cuza Iaşi. Mat. (N.S.), 57 (2011), 65-74.

[4] F. S. De Blasi, J. Myjak, Sur la porosite de l'ensemble des contractions sans point fixe, (French) [[On the porosity of the set of contractions without fixed points]] C. R. Acad. Sci. Paris Sér. I Math., 308 (1989), 51-54. 1

[5] R. Kannan, Some results on fixed points, II, Amer. Math. Monthly, 76 (1969), 405-408. 1, 1.2

[6] B. K. Lahiri, P. Das, Well-posedness and porosity of a certain class of operators, Demonstratio Math., 38 (2005), 169-176. 1

[7] N. M. Mlaiki, A contraction principle in partial S-metric spaces, Univers. J. Math. Math. Sci., 5 (2014), 109-119. 1, 1.5 
[8] N. M. Mlaiki, N. Souayah, K. Abodayeh, T. Abdeljawad, Contraction principles in $\mathrm{M}_{\mathrm{s}}$-metric spaces, J. Nonlinear Sci. Appl., 10 (2017), 575-582. 1, 1.3, 1.4, 1.7

[9] A. Pansuwan, W. Sintunavarat, J. Y. Choi, Y. J. Cho, Ulam-Hyers stability, well-posedness and limit shadowing property of the fixed point problems in M-metric spaces, J. Nonlinear Sci. Appl., 9 (2016), 4489-4499. 1

[10] S. Reich, A. J. Zaslavski, Well-posedness of fixed point problems, Far East J. Math. Sci. (FJMS), Special Volume, Part III, (2001), 393-401. 1

[11] S. Sedghi, N. Shobe, A. Aliouche, A generalization of fixed point theorems in S-metric spaces, Mat. Vesnik, 64 (2012), 258-266. 1, 1.13

[12] P. V. Subrahmanyam, Completeness and fixed-points, Monatsh. Math., 80 (1975), 325-330. 1 